\title{
Progress on an open source computer-assisted structure elucidation suite (SENECA)
}

\author{
Stefan Kuhn*, Christoph Steinbeck \\ From 5th German Conference on Cheminformatics: 23. ClC-Workshop \\ Goslar, Germany. 8-10 November 2009
}

We suggested and developed various components for Computer-Assisted Structure Elucidation (CASE) over the years $[1,2]$. Our current goal is to integrate these into an easy to use and efficient platform for end users, called SENECA. This is based on Bioclipse [3], an integrated software suite for chemo- and bioinformatics providing plugins for file handling and visualisation of compounds and spectra.

The SENECA feature currently comprises the following components and algorithms:

- A deterministic structure generator suitable for small chemical spaces.

- Simulated Annealing and Genetic Algorithm for random structure walks in large spaces.

- Simulation of 13C NMR spectra for ranking results.

SENECA is a Bioclipse feature, available via the update site at http://www.ebi.ac.uk/steinbeck-srv/speclipse/net. bioclipse.seneca-updatesite/.

Published: 4 May 2010

\section{References}

1. Steinbeck C, Krause S, Kuhn S: NMRShiftDB - Constructing a free chemical information system with open-source components. Journal of Chemical Information and Computer Sciences 2003, 43:1733-1739.

2. Kuhn S, Egert B, Neumann S, Steinbeck C: Building blocks for automated elucidation of metabolites: Machine learning methods for NMR prediction. BMC Bioinformatics 2008, 9:400.

3. Spjuth O, Helmus T, Willighagen EL, Kuhn S, Eklund M, et al: Bioclipse: An open source workbench for chemo- and bioinformatics. BMC Bioinformatics 2007, 8:59.

\section{doi:10.1186/1758-2946-2-S1-P34}

Cite this article as: Kuhn and Steinbeck: Progress on an open source computer-assisted structure elucidation suite (SENECA). Journal of Cheminformatics 2010 2(Suppl 1):P34.

Wellcome Trust Genome Campus, Hinxton, Cambridge CB10 1SD, UK

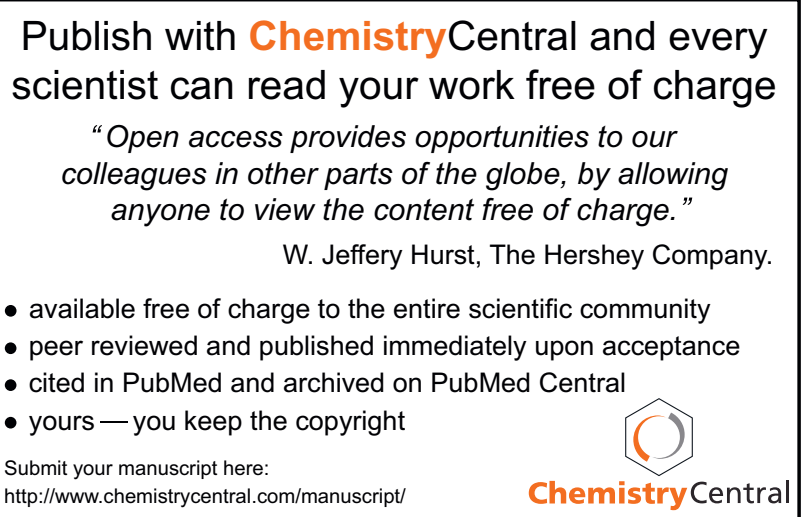

